\title{
Falling short: examination of the validity of methods used to identify paediatric hospital falls in NSW, Australia
}

\section{Daniela Feuerlichta,g, Maria Agaliotis ${ }^{b, c}$ and Reece Hinchcliffb,d,e,f}

a The Sydney Children's Hospitals Network, NSW, Australia

b School of Public Health and Community Medicine, UNSW Sydney, Australia

c Australian Institute of Health Service Management, Tasmanian School of Business and Economics, University of Tasmania, Sydney, NSW

d School of Public Health and Social Work, Faculty of Health, Queensland University of Technology, Brisbane, Australia

e Faculty of Health, Centre for Health Services Management, University of Technology Sydney, NSW, Australia

f Australian Institute of Health Innovation, Faculty of Medicine and Health Sciences, Macquarie University, Sydney, NSW

g Corresponding author: Daniela.Feuerlicht@health.nsw.gov.au

\section{Article history}

Publication date: March 2020

Citation: Feuerlicht D, Agaliotis M, Hinchcliff R. Falling short: examination of the validity of methods used to identify paediatric hospital falls in NSW, Australia. Public Health Res Pract. 2020;30(1):e29121907. First published: 2 May 2019. https://doi.org/10.17061/ phrp29121907

\section{Key points}

- This article provides previously unavailable data on the frequency, level of harm and demographics of paediatric falls in two Australian hospitals

- Information identified in this study is already being used to further develop paediatric falls prevention resources in New South Wales

- A similar methodology can be used to provide more robust data on other patient safety issues. The information obtained through this process can be used to inform patient safety initiatives

\section{Abstract}

Objective: Hospital falls are a major cause of costly harm. This descriptive epidemiological study reports the results of a data linkage and medical record review to examine the relative utility of these methods for identifying paediatric in-hospital falls.

Type of program or service: The study was conducted across two tertiary and quaternary specialist paediatric hospitals in New South Wales (NSW), Australia.

Methods: A retrospective audit was conducted of paediatric falls occurring in hospital between 1 July 2015 and 30 June 2016. Falls were identified using two systems: the Incident Information Management System (IIMS) and medical records coded data (MRCD). These data were linked, and falls were verified using medical record review.

Results: Of the 146 in-hospital falls identified, only 28 (19\%) were included in both systems. The IIMS identified 137 falls, with five excluded. The MRCD identified 63 admissions in which falls occurred, with 27 excluded, and screening of clinical notes found 42 falls in the remaining 36 admissions.

Lessons learnt: There was a discrepancy in the number of falls identified in the two administrative datasets due to different inclusion criteria. The medical record review provided additional practice-relevant information that was unavailable within the two datasets. This descriptive epidemiological study highlights the value of combining data linkage with medical record review, but also the need for more critical reflection on the methods used to collect and report falls data in Australian hospitals, so that comprehensive and accurate data can be used to inform quality and safety interventions.

This analysis will inform improvements to data capture methods and provide data to advise paediatric falls prevention strategies within the NSW paediatric hospital context. 


\section{Background}

Hospital falls are recognised as a major cause of costly harm, both in Australia ${ }^{1}$ and globally. ${ }^{2}$ In 2015, an Australian multisite prospective cohort study ${ }^{1}$ found that falls in adult hospital patients resulted in a mean increase in length of stay of 8 days, and a mean increase in costs of $\$ 6669$. The specific cost of falls in the paediatric context has not been evaluated.

In-hospital falls can result in harm to patients. A multisite American study identified that injury occurred in almost one-third of in-hospital paediatric falls over a 6-month period. Although the falls identified in this study rarely increased hospital length of stay, almost half of falls were found to be preventable. ${ }^{3}$ Decreasing the burden of in-hospital falls across all ages is a national priority in Australia. The Australian Commission on Safety and Quality in Health Care's National Safety and Quality Health Service Standards (second edition) identifies specific criteria for hospitals to reduce risk and harm from falls. ${ }^{4}$

In New South Wales (NSW), data on falls are routinely captured through the Incident Information Management System (IIMS) and medical records coded data (MRCD). The IIMS is the incident reporting system used in NSW. It was established as a key component of the NSW Patient Safety and Clinical Quality Program ${ }^{5}$, to identify incidents that occur in the hospital environment that can inform patient safety strategies. MRCD are captured by clinical coders using the ICD-10-AM (International Statistical Classification of Diseases and Related Health Problems, 10th revision, Australian modification $)^{6}$ and are used for hospital funding, research, education, planning and clinical benchmarking.

Both administrative datasets have demonstrated limitations in collecting falls data. ${ }^{7}$ Coding of falls by clinical coders requires accurate documentation by staff. Incident reporting requires staff to enter incidents into a reporting system. Barriers to reporting include time constraints and inadequate feedback to reporting staff. ${ }^{8}$ Staff perceptions of reporting paediatric in-hospital falls have not been explored.

Data linkage provides a potentially useful strategy to generate more reliable information from the IIMS and MRCD datasets. ${ }^{9}$ In addition, medical record review provides quality assurance for routine incident reporting. ${ }^{10}$ A previous study ${ }^{11}$ in NSW linked the IIMS and MRCD datasets in the adult population, but did not review medical records. There are no published studies where the IIMS and MRCD datasets have been linked to examine the quality of paediatric hospital falls data in the Australian context.

The aim of this study was to perform data linkage using existing administrative datasets - IIMS and MRCD - complemented by medical record review, to critically examine the information provided from the two datasets and collect falls data. This analysis will inform improvements to data capture methods and provide data to advise paediatric falls prevention strategies within the NSW paediatric hospital context.

\section{Setting}

The study was conducted across two tertiary and quaternary specialist paediatric hospitals in NSW, Australia. The two hospitals have more than 50000 inpatient admissions each year. Falls prevention strategies at these hospitals include screening all inpatients using a falls risk assessment, the modified Humpty Dumpty Falls Scale ${ }^{12}$, which identifies patients as being at either low or high risk of falls. All hospital staff are required to notify identified incidents in the IIMS. Local management and committees review incidents and trends. Aggregated IIMS data across NSW are also monitored by state-wide government agencies.

For this study, in-hospital falls identified in patients aged 0 to 18 years occurring between 1 July 2015 and 30 June 2016 were analysed. Admissions with ICD-10AM falls (W00-W19) and health service area (Y92.22) codes were identified and extracted from the MRCD. Incidents that identified 'fall' as one of the incident types were extracted from the IIMS. The first author screened all clinical notes for identified falls and obtained additional information from the medical record. Falls that occurred outside the hospital setting were excluded, as were falls that did not meet the World Health Organization (WHO) definition of a fall as "an event which results in a person coming to rest inadvertently on the ground or floor or other lower level". ${ }^{13}$ Falls were also classified as 'developmental' (i.e. occurring due to immature gross motor skills) or 'other', to allow comparisons with international literature. ${ }^{14}$

Records were linked using patients' unique medical record numbers and dates of admission. The total admission numbers were obtained from the hospital analysis unit. Descriptive analysis was carried out using International Business Machines Statistical Package for the Social Sciences (Armonk, NY: IBM Corp; version 23). Ethics approval was obtained through the Human Research Ethics Committee responsible for both hospitals (LNR/16/SCHN/267).

\section{Outcomes}

The rate of all inpatient falls was 2.92 per 1000 admissions, or 0.94 per 1000 bed days. When falls due to developmental issues were excluded (to allow comparison with international literature ${ }^{14}$ ), the rate of falls was 0.88 per 1000 bed days. The IIMS identified 137 falls, with five excluded, based on the inclusion criteria. The MRCD identified 63 admissions where falls occurred, with medical record review confirming that 36 of these admissions met the inclusion criteria. The same medical record review identified that 42 falls occurred in these 36 admissions. Therefore, in total, 146 falls met the inclusion criteria - 132 in the IIMS and 42 in the MRCD with 28 falls recorded in both systems. Just over half the patients were male (Table 1). 
Table 1. Frequency distribution of falls in different developmental age groups

\begin{tabular}{|c|c|c|c|}
\hline \multirow[b]{2}{*}{ Age group (years) } & \multicolumn{2}{|c|}{ Gender $n(\%)$} & \multirow{2}{*}{$\begin{array}{l}\text { Total } \\
n(\%)\end{array}$} \\
\hline & Male & Female & \\
\hline$<3$ & $34(24.6)$ & $16(11.6)$ & $50(36.2)$ \\
\hline 3 to $<7$ & 15 (10.9) & $12(8.7)$ & 27 (19.6) \\
\hline 7 to $<13$ & $14(10.1)$ & $10(7.2)$ & $24(17.4)$ \\
\hline 13 to 18 & $11(8.0)$ & $26(18.8)$ & $37(26.8)$ \\
\hline All ages & $74(53.6)$ & $64(46.4)$ & $138(100)$ \\
\hline
\end{tabular}

a Excludes eight falls where gender was not specified

Of the 146 falls identified, the outcome of the fall was identified in 135 cases. Fifty-eight (43\%) patients suffered some level of harm, including four who had fractures. IIMS identified 47 falls that resulted in harm (47/132; $36 \%$ ), while MRCD identified 26 falls that resulted in harm (26/42; 62\%). A total of 84 patients were screened for their falls risk before their fall, and, of these, 15 (18\%) were identified to be at high risk of a fall. The majority of falls occurred among inpatients (106, or 76\%) (Table 2). Almost half the falls (66, or $45 \%$ ) occurred at the patient's bedside. Parents or carers were present during 82 (56\%) of the 146 falls, while healthcare workers were present in $29(20 \%)$ cases.

Table 2. Frequency of fall by patient type

\begin{tabular}{lc}
\hline Patient type & $\begin{array}{c}\text { Frequency } \\
n(\%)\end{array}$ \\
\hline Inpatient & $106(75.7)$ \\
Emergency & $21(15)$ \\
Outpatient & $13(9.3)$ \\
Total $^{\text {b }}$ & 140 \\
\hline
\end{tabular}

b Excludes six patients where patient type could not be identified

\section{Limitations}

Neither the IIMS nor MRCD identified all the falls in this study. This can be partly attributed to differing definitions of falls used in the two datasets. All falls, as well as incidents where a fall nearly occurred, should be reported in the IIMS. For MRCD, only a fall that results in additional care or treatment for an inpatient is coded.

Codes used by the MRCD are only used for inpatients and are only assigned once per admission, irrespective of the number of falls that occur during the admission. In addition, the methodology described will extract admissions where a fall has occurred outside the hospital, and the health service area code (Y92.22) is assigned for another reason during the admission. This latter explanation would account for the large number of MRCD admissions that were excluded $(n=27)$.

\section{Discussion}

This epidemiological study of paediatric falls was the first of its kind to be undertaken in an Australian paediatric setting. The rate of paediatric falls reported across the two hospitals ( 0.88 per 1000 bed days) is comparable with that reported for paediatric populations internationally. Kingston et al. studied a network of paediatric 'Magnet' hospitals and identified a falls rate of 0.87 per 1000 bed days. ${ }^{14}$ Hospitals that receive the American Nurses Credentialing Center Magnet recognition achieve above the mean on a number of nurse-sensitive outcome measures, including prevention of hospital falls. ${ }^{15}$

Paediatric in-hospital falls can cause serious harm. As identified in this study, $43 \%$ of patients suffered some level of injury, with four patients experiencing fractures. This rate of injury is higher than reported in international literature; ${ }^{3}$ however, the identification of injury may be subjective, and the data collection methods differed between the two studies. A recent publication from the Independent Hospital Pricing Authority ${ }^{16}$ identified that for admissions in which an in-hospital fall resulted in a fracture or other intracranial injury, the cost incurred was, on average, $2.5 \%$ higher than for similar admissions without a fall.

Falls prevention strategies include identifying risk and providing education. This study found that only $18 \%$ of patients who had their falls risk assessed before experiencing falls were classified as being at high risk. Further investigation is warranted to determine if this low proportion is representative of the general paediatric hospital population. In this study, falls frequently occurred at the patient's bedside and in the company of parents or carers, so this factor should be considered when targeting future education.

Our study demonstrated that falls identified in MRCD had a higher proportion of harm, compared to those identified through the IIMS. This can be attributed to the differing definitions of falls as outlined earlier. Neither system captured all the falls identified in the study. Policies, research and other patient safety initiatives that rely on either system should be interpreted with caution, even if data linkage has occurred. 
Medical record review provided additional evidence to identify trends and factors related to hospital falls. The data collected during this study have been incorporated into the development of state-wide paediatric falls prevention resources. ${ }^{17}$ This verifies the utility, and potential for broader application, of the methods used. More comprehensive data repositories will enable identification of the frequency and burden of patient safety risks, to further guide prevention strategies.

\section{Conclusion}

This epidemiological study of paediatric falls in Australian hospitals highlights the need for more critical reflection on methods that are currently used to collect and report falls data in Australian hospitals. This study demonstrates the value of combining data linkage with medical record review to collect data that informs quality and safety interventions - as evidenced by the use of data collected in this study in the development of state-wide paediatric falls prevention resources. ${ }^{17}$

The methods used have potential for broader application in healthcare settings to provide more robust data. More comprehensive data repositories will enable improved identification of patient safety risks to help guide prevention strategies.

\section{Acknowledgements}

We thank Chrissy Ceely and Kate Butler for their assistance in the formation and analysis of the study.

\section{Peer review and provenance}

Externally peer reviewed, not commissioned.

\section{Competing interests}

None declared.

\section{Author contributions}

DF designed the study, collected, linked and analysed the data and drafted the manuscript. MA assisted in reviewing the data analysis and reviewed the manuscript. $\mathrm{RH}$ assisted in developing the study design and critically reviewed the manuscript.

\section{References}

1. Morello RT, Barker AL, Watts JJ, Haines T, Zavarsek SS, Hill KD, et al. The extra resource burden of in-hospital falls: a cost of falls study. Med J Aust. 2015;203(9):367.
2. NHS Improvement. The incidence and costs of inpatient falls in hospitals. London: NHS Improvement; 2017 [cited 2018 Jan 28]. Available from: improvement.nhs.uk/ documents/1471/Falls_report_July2017.v2.pdf

3. Jamerson PA, Graf E, Messmer PR, Fields HW, Barton S, Berger A, et al. Inpatient falls in freestanding children's hospitals. Pediatr Nurs. 2014;40(3);127-35.

4. Australian Commission on Safety and Quality in Health Care. Sydney: ACSQHC; 2019. Assessment to the NSQHS standards; [cited 2018 Jan 28]. Available from: www.safetyandquality.gov.au/our-work/assessment-to-thensqhs-standards/

5. NSW Health. NSW Patient Safety and Clinical Quality Program. Sydney: NSW Health, Clinical Excellence Commission; 2005 [cited 2018 Apr 2]. Available from: www1.health.nsw.gov.au/pds/ActivePDSDocuments/ PD2005_608.pdf

6. Australian Consortium for Classification Development. International statistical classification of diseases and related health problems, tenth revision, Australian modification (ICD-10-AM/ACHI/ACS). Sydney: Independent Hospital Pricing Authority; 2017 [cited 2018 Jan 28]. Available from: www.accd.net.au/lcd10.aspx

7. Hill AM, Hoffmann T, Hill K, Oliver D, Beer C, McPhail S, et al. Measuring falls events in acute hospitals $-\mathrm{a}$ comparison of three reporting methods to identify missing data in the hospital reporting system. J Am Geriatr Soc. 2010;58(7):1347-52.

8. Kingston MJ, Evans SM, Smith BJ, Berry JG. Attitudes of doctors and nurses towards incident reporting: a qualitative analysis. Med J Aust. 2004;181(1):36-9.

9. Jutte DP, Roos LL, Brownell MD. Administrative record linkage as a tool for public health research. Annu Rev Public Health. 2011;21(32):91-108.

10. Sari AB, Sheldon TA, Cracknell A, Turnbull A. Sensitivity of routine system for reporting patient safety incidents in an NHS hospital: retrospective patient case note review. BMJ. 2007;334(7584):79.

11. Trinh LTT, Achat $H$, Assareh $H$. Use of routinely collected data in reporting falls in hospitals in a local health district in New South Wales, Australia. Health Inf Manag. 2017;46(1):15-22.

12. Hill-Rodriguez D, Messmer PR, Williams PD, Zeller RA, Williams AR, Wood M, Henry M. The Humpty Dumpty Falls Scale: a case-control study. J Spec Pediatr Nurs. 2009;14(1):22-32.

13. World Health Organization. Geneva: WHO; 2019. Falls: key facts; 2018 Jan 16 [cited 2018 Jan 28]; [about 3 screens]. Available from: www.who.int/mediacentre/factsheets/fs344/en/

14. Kingston F, Bryant T, Speer K. Pediatric falls benchmarking collaborative.

J Nurs Adm. 2010;15(4):261-2. 
15. Graf E. Magnet children's hospitals: leading knowledge development and quality standards for inpatient pediatric fall prevention programs. J Pediatr Nurs. 2011;26(2):122-7.

16. Independent Hospital Pricing Authority. Pricing and funding for safety and quality: risk adjustment model for hospital acquired complications, version 3. Sydney: IHPA; 2018 [cited 2019 Mar 12]. Available from: www.ihpa.gov. au/sites/default/files/publications/pricing_and_funding_ for_safety_and_quality_-_risk_adjustment_model_for_ hospital_acquired_complications_2018-19.pdf
17. Clinical Excellence Commission. Sydney: Clinical Excellence Commission. Paediatric patient safety: falls prevention; 2018 [cited 2018 Feb 18]; [about 3 screens]. Available from: www.cec.health.nsw.gov.au/patientsafety-programs/paediatric-patient-safety/pqp-fallsprevention

\section{Copyright: (c) (i) (2)}

(C) 2019 Feuerlicht et al. This article is licensed under the Creative Commons Attribution-NonCommercial-ShareAlike 4.0 International Licence, which allows others to redistribute, adapt and share this work non-commercially provided they attribute the work and any adapted version of it is distributed under the same Creative Commons licence terms. See: www.creativecommons.org/licenses/by-nc-sa/4.0/ 